\title{
Investigation of Radiation Effects in Sprouts of Barley
}

N. Shamal and V. Knatko

Institute of Radiobiology of NAS of Belarus, Fedjuninskogo, 4, 246007 Gomel, Belarus nikitsin@tut.by

The radioactive environmental contamination caused by Chernobyl accident, stimulated working out of a problem of radiation protection biota. At the present investigation the following reactions of plants to irradiation have been chosen: germination of seeds, a state of mitotic cells of roots, phototaxis of chloroplasts and parity of chlorophyll forms in vivo. The research object was barley. The doses of irradiation were 2.5 and $5.0 \mathrm{~Gy}$. According to the results, indicators of germination of seeds obtained for the irradiated and not irradiated objects have been appeared almost identical $(\sim 94 \%)$. The analysis of cells of roots has revealed acceleration of rates of cell fission at the irradiated plants in comparison with not irradiated plants. The dependences of damage cells and of exit of aberration cells from irradiation dose were observed. Phototaxis of chloroplast was estimated for 7-day sprouts. Phototaxis (moving chloroplast under the influence of light) - the normal physiological process occurring in cells of leaves. At the present study the decrease negative and positive phototaxis at the irradiated plants has been established. Dependence between decrease in intensity of this process and an irradiation dose is observed. Positive phototaxis has decreased in 1.4 and 1.8 times, accordingly at an irradiation in a dose 2.5 and 5.0 Gy, negative - in 1.2 and 1.7 times, respectively. The definition of the maintenance of chlorophyll forms (range of 650$715 \mathrm{~nm}$ ) in the 1st leave of the 8- and 11-days sprouts of the barley which has grown from seeds irradiated in dose 4, 0 Gy. Changes of percentage of four forms at 8-days and two forms at 11-days plants were established. At 8-days sprouts the increase in a share of spectral forms of 671 and $683 \mathrm{~nm}$ on 7.5 and $14.1 \%$, accordingly, and decrease in forms of 686 and $693 \mathrm{~nm}$ on 14.5 and $7.1 \%$ was observed. In case of 11-days sprouts the distinction in the maintenance of spectral forms of 671 and $693 \mathrm{~nm}$ smooth out, for the form of $683 \mathrm{~nm}$ practically remain $(12.9 \%)$ and for the form of $686 \mathrm{~nm}$ increase to $58.6 \%$. The presented results together with future data for smaller doses can used to obtain the dose-effect relationship. 